\title{
Planarization of patterned magnetic recording media to enable head flyability
}

\author{
Chulmin Choi $\cdot$ Yeoungchin Yoon • \\ Daehoon Hong $\cdot$ Young Oh $\cdot$ Frank E. Talke • \\ Sungho Jin
}

Received: 16 September 2010/ Accepted: 4 January 2011/Published online: 29 January 2011

(C) The Author(s) 2011. This article is published with open access at Springerlink.com

\begin{abstract}
The fabrication and planarization of patterned magnetic recording media is investigated and the flyability of magnetic recording sliders on a patterned and planarized $65 \mathrm{~mm}$ glass disk is investigated a small coupon of patterned media with an array of nano pillars of $40 \mathrm{~nm}$ diameter and $60 \mathrm{~nm}$ height was first fabricated by e-beam lithography and reactive ion etching (RIE) to investigate the planarization process for patterned media. Since read/ write flyability tests require a patterned disk rather than a small coupon area, we have prepared a bit patterned glass disks of $65 \mathrm{~mm}$ diameter (2.5 in.) using the so-called " $\mathrm{Ag}$ ball-up process" in combination with RIE. This "Ag ballup process" permits the manufacturing of a nano-sized bit patterns on a large area, i.e., on a disk with $65 \mathrm{~mm}$ diameter. Planarization of the patterned area was performed with hydrogen silsesquioxane (HSQ) by spin coating. The HSQ layer was back-etched using RIE, resulting in a smooth surface. "Flyability testing" indicates significantly improved flying stability of typical magnetic recording sliders on the planarized glass disks, with the standard deviation of flying height fluctuations on the order of
\end{abstract}

C. Choi · Y. Yoon · D. Hong · Y. Oh · F. E. Talke · S. Jin ( $\square)$

Center for Magnetic Recording Research,

University of California at San Diego,

La Jolla, CA 92093, USA

e-mail: jin@ucsd.edu

C. Choi $\cdot$ Y. Oh $\cdot$ S. Jin

Materials Science and Engineering, University of California at San Diego, La Jolla, CA 92093, USA

Y. Yoon - D. Hong $\cdot$ F. E. Talke $\cdot$ S. Jin

Mechanical and Aerospace Engineering,

University of California at San Diego,

La Jolla, CA 92093, USA
$0.1 \mathrm{~nm}$. The latter value is comparable to that of "smooth" disks.

\section{Introduction}

Bit patterned recording media (BPM) have received increased attention as potential candidates for areal densities in excess of $1 \mathrm{Tbit} / \mathrm{in}^{2}$. (Richter et al. 2006; Terris and Thomson 2005). As the areal density in disk drives increases to values near $1 \mathrm{Tbit} / \mathrm{in} .^{2}$, the magnetic energy of a single bit is not sufficient anymore to prevent thermally activated bit reversals, and errors in the read back process are likely to occur. For bit patterned media (BPM), the switching volume is identical to that of an individual bit since the size of the bits is lithographically pre-defined. The size of a typical bit in BPM has linear dimensions as small as $10 \mathrm{~nm}$. This small bit is expected to be stable with respect to thermal fluctuations, have a sharp magnetic transition and exhibit low noise, both along and across a track.

Two major fabrication methods exist for BPM (Thomson et al. 2006; Shaw et al. 2007; Kitade et al. 2004). In the first method, a layer of high coercivity magnetic material is first deposited on a flat substrate. This layer is then patterned into discrete magnetic bits using lithographic techniques such as ion beam milling, reactive ion etching (RIE), or focused ion beam milling (FIB). The main advantage of this method is that no magnetic material is left between neighboring bits to cause magnetic interference. In addition, roughness on the media surface is uniform because the height of the bits is identical to the thickness of the recording layer. However, a main disadvantage of this method is that the magnetic material can easily be damaged by the ion etching patterning process. Furthermore, material removed during ion-etching can be 
re-deposited on the media during the patterning process, which is undesirable (Shaw et al. 2007; Kitade et al. 2004). In the second method, the substrate is lithographically prepatterned into islands, or so-called pillars, before the magnetic material is deposited. The advantage of this method is that the magnetic material deposited on the top of the pillars is isolated from adjacent islands without the need for ion milling or other etching methods, i.e., there is no damage of the magnetic material due to processing of the pattern. However, the magnetic material in the areas adjacent to bits (trenches) can introduce undesirable noise during the read/write process and magnetic interactions between the magnetic materials deposited in the trench areas and on the top of the islands.

The RIE to remove the overfilled HSQ resist is performed using oxygen ions. Unlike the Ar atoms used for physical ion milling and removal of magnetic metal layer for patterning, the oxygen ion bombardment is much milder and hence the magnetic recording layer is not damaged. Furthermore, the top layer of the $\mathrm{Co} / \mathrm{Pd}$ multilayer material to be patterned is the Pd layer, which does not react with oxygen. Therefore, the magnetic property is not affected much during the oxygen RIE process.

In order to minimize magnetic interactions of this latter type, the height of the individual pillars should be large enough so that the magnetic material deposited in the trench areas does not contribute much to the read signal picked up by the flying read/write head. The desirable height would depend on the diameter of the bit pattern as well as the spacing between the adjacent bits. The pillar aspect ratio of 1:1 or higher would be desirable. However, protruding pillars of large height exert a significant influence on the flying characteristics of magnetic heads. In fact, investigations on discrete track media disks have shown that flying of a slider over a discrete track disk may not be possible at all if the flying height loss due to the discrete tracks exceeds the design flying height of the slider on a smooth disk. The loss of flying height of a slider on BPM is anticipated to be more severe with an increase in the height (depth) of the bit pattern. To reduce topographical effects of the bit pattern (pillar height), it is necessary to fill the recessed areas with a nonmagnetic material to create a "smooth" disk. The process of filling the trench regions around protruding bits is called "planarization". It has been reported by Hattori et al. (Hattori et al. 2004) that deposition of $\mathrm{SiO}_{2}$ by sputtering and removal of the excess $\mathrm{SiO}_{2}$ by chemical mechanical polishing (CMP) is an effective method for planarization of BPM. However, CMP is delicate and time-consuming, and damage of magnetic material could easily occur during the mechanical/chemical polishing process.

In this paper we demonstrate a simple and efficient planarization method for BPM surfaces containing nano- scale surface topography. We use hydrogen silsesquioxane (HSQ), a commonly used negative resist, to fill and planarize the nano-topographically patterned recording media, which on annealing converts to mostly silicon oxide. The HSQ can easily be spin-coated to fill the etched recess areas. In addition, the mechanical properties and thickness of HSQ can be controlled for optimum "slider flyability" by altering and/or controlling the post-deposition baking temperature and plasma treatment. After spin-coating, HSQ is back-etched by RIE to remove most of the excess material beyond the top surface of the magnetic bits. Flyability tests with planarized BPM were performed, which demonstrated excellent flying characteristics.

\section{Experimental procedures}

Figure 1 shows the schematic of fabrication of BPM using e-beam lithographic and planarization with HSQ (Dow Corning No. Fox-12). First, HSQ solution was spin-coated on a $\mathrm{Si}$ substrate as a $40 \mathrm{~nm}$ thick layer and baked on a hotplate. Then, e-beam exposure was carried out with an Raith 50 E-beam writer (Fig. 1b). Thereafter, the HSQ resist was developed in a tetramethylammonium hydroxide (TMAH) aqueous solution at room temperature (Fig. 1c). After HSQ resist development, the Si substrate with patterned HSQ was etched in a gas mixture of C4F8 and SF6 using RIE (Oxford Plasmalab-100) for pattern transfer into the substrate (Fig. 1d). After the dry-etching step, the HSQ mask was removed (Fig. 1e) and a magnetically perpendicular anisotropic hard $\mathrm{Co} / \mathrm{Pd}$ multilayer film with a \{Ta $3 \mathrm{~nm} \backslash P d \quad 3 \mathrm{~nm} \backslash[\text { Co } 0.3 \mathrm{~nm} \backslash \mathrm{Pd} 0.8 \mathrm{~nm}]_{8}$ \} structure was sputtered on the pre-patterned substrate (Fig. 1f). The coercive force of the $\mathrm{Co} / \mathrm{Pd}$ multilayer was $\sim 3,000 \mathrm{Oe}$, an appropriate value for magnetic recording. A HSQ film was then spun on the patterned media to fill the "trenches" (Fig. 1g) and subsequently re-etched by RIE to remove the "overfill" regions on the disk (Fig. 1h). The HSQ material gets preferentially RIE etched as compared to the magnetic recording material, and hence the degree of planarization can be controlled at will with RIE time. A flush surface, slightly recessed, or significantly recessed configuration can be obtained as discussed later for flying height evaluations on intentional variation of the degree of recessed geometry. The disk was then baked on a hotplate at $110^{\circ} \mathrm{C}$ for $180 \mathrm{~s}$ to vaporize the solvent in HSQ. The HSQ was mechanically hardened by oxygen plasma irradiation using radio frequency (RF) RIE (Fig. 1i). This hardening process, which does not noticeably affect the HSQ thickness, is added for the purpose of preventing a soft polymer filling material from getting mechanically damaged and contaminating the surface of flying head, and also to minimize the chance of head crash and flying height reduction. 
Fig. 1 Schematic of fabrication of bit patterned media (BPM) using e-beam lithography and the method for planarization with hydrogen silsesquioxane (HSQ)

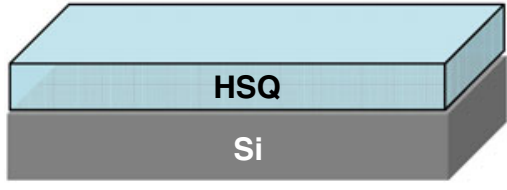

(a) Spin-coating HSQ Resist

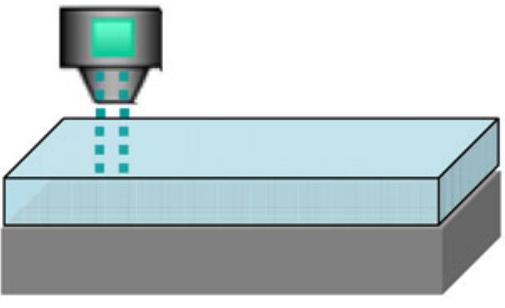

(b) Expose by E-beam

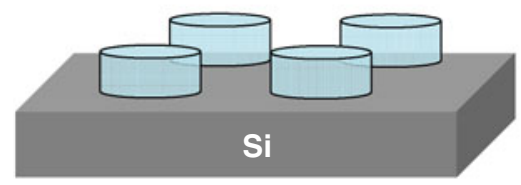

(c) Development

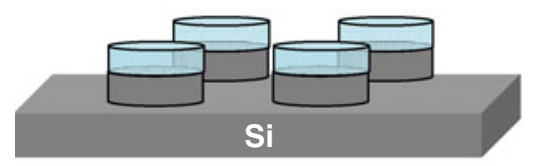

(d) Reactive lon Etching

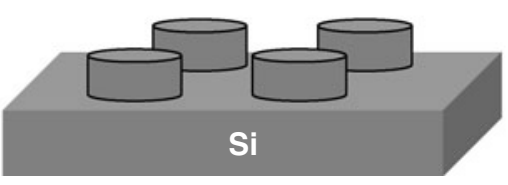

(e) Removal of HSQ mask

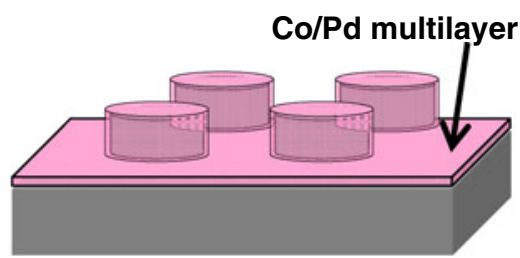

(f) Deposition of magnetic materials by sputtering

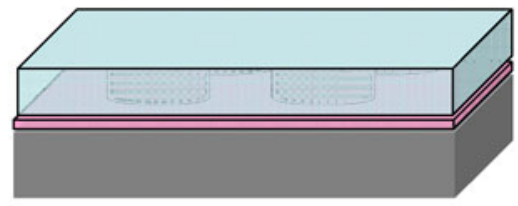

(g) Filling trenches with HSQ by spin coating

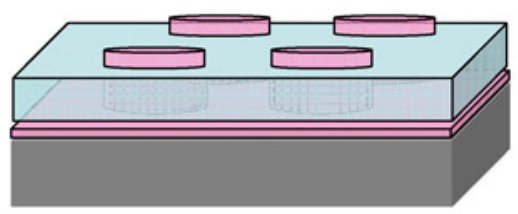

(h) Removal of overfilled HSQ by RIE

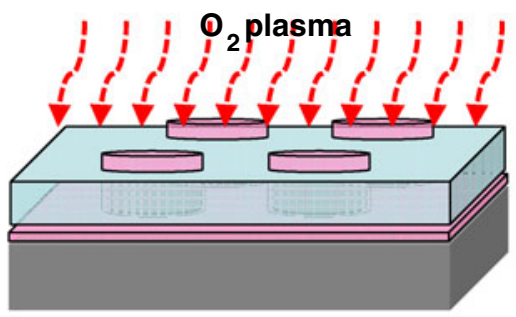

(i) Hardening of HSQ by oxygen plasma
The gas flow rate, pressure, and RF power used for the RIE were $50 \mathrm{sccm}, 100 \mathrm{mTorr}$, and $300 \mathrm{~W}$, respectively.

The morphology of the BPM on a Si substrate was visualized using field emission scanning electron microscopy (SEM) and atomic force microscopy (AFM) before and after planarization.

In order to produce patterned media on a large area required for flyability tests, we prepared a pattern of protruding pillars on a $65 \mathrm{~mm}$ (2.5 in.) glass disk substrate using the following method. Rather than exposing the disk using e-beam lithography, we sputter deposited a thin film of $\mathrm{Ag}$ on the disk, followed by annealing in vacuum at temperatures of up to $650^{\circ} \mathrm{C}$. This step caused the break up of the film into small islands which are comparable in average size to the nano-pillar diameter fabricated using e-beam lithography. In this paper we denote the break-up process as the "ball-up process" because of the spheroidization type change of film morphology caused by the break-up of the continuous film (Fig. 2a). The small Ag nano islands were then used as an etch mask. RIE with a gas mixture of $\mathrm{CHF}_{3}$ and $\mathrm{Ar}$ (Oxford Plasmalab-80) was subsequently performed to create the bit patterns onto the full glass disk surface (Fig. 2b). After RIE, the Ag islands were chemically etched away with $\mathrm{Ag}$ etchant $\left(\mathrm{HNO}_{3}: \mathrm{H}_{2} \mathrm{O}=1: 1\right)$. The subsequent process steps used after this step were exactly the same as those used for planarization of e-beam patterned material with HSQ. Prior to flyability testing, a diamond like carbon (DLC) film of $5 \mathrm{~nm}$ was sputtered on the planarized bit patterned media (BPM) and a thin lubricant film (Zdol 2000) was applied by 


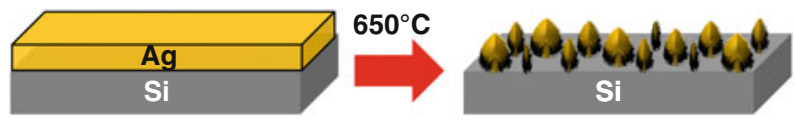

(a) Ball-up process

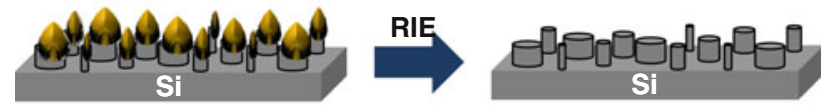

(b) RIE and Removal of Ag mask

(c) Deposition of magnetic materials by sputtering

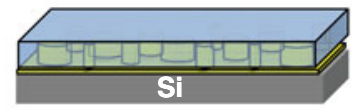

(d) Filling trenches with HSQ by spin coating

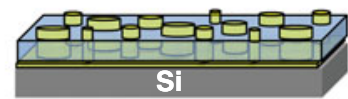

(e) Removal of overfiliiedHSQ by RIE

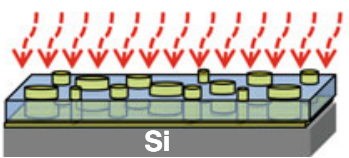

(f) Hardening of HSQ by oxygen plasma

Fig. 2 Schematic of fabrication of bit patterned media (BPM) using $\mathrm{Ag}$ "ball-up" method and the method for planarization with hydrogen silsesquioxane (HSQ)

dip coating, similar to the dip coating step in the manufacturing process of hard disks. The head flyability was evaluated before and after planarization.

\section{Results and discussion}

Periodic nano pillar patterns on small coupons were fabricated using e-beam lithography as described in Fig. 1. In this paper, we demonstrated periodic nano pillars with an island diameter of $\sim 40 \mathrm{~nm}$ using negative e-beam resist (HSQ). In Fig. 3a an oblique angle SEM image is shown of the periodic nano pillar pattern fabricated by e-beam lithography. The protruding Si islands in Fig. $3 b$ have a diameter of approximately $40 \mathrm{~nm}$ and heights of $60 \mathrm{~nm}$, corresponding to an equivalent areal bit density of $\sim 0.15$ Tbit/in. ${ }^{2}$. A higher-density pattern with a $10 \mathrm{~nm}$ diameter bit array (1.6 Tbit/in. $\left.{ }^{2}\right)$ was also fabricated (Fig. 3b).

In Fig. 4, SEM and AFM images are shown of a BPM on a $\mathrm{Si}$ substrate fabricated by e-beam lithography.
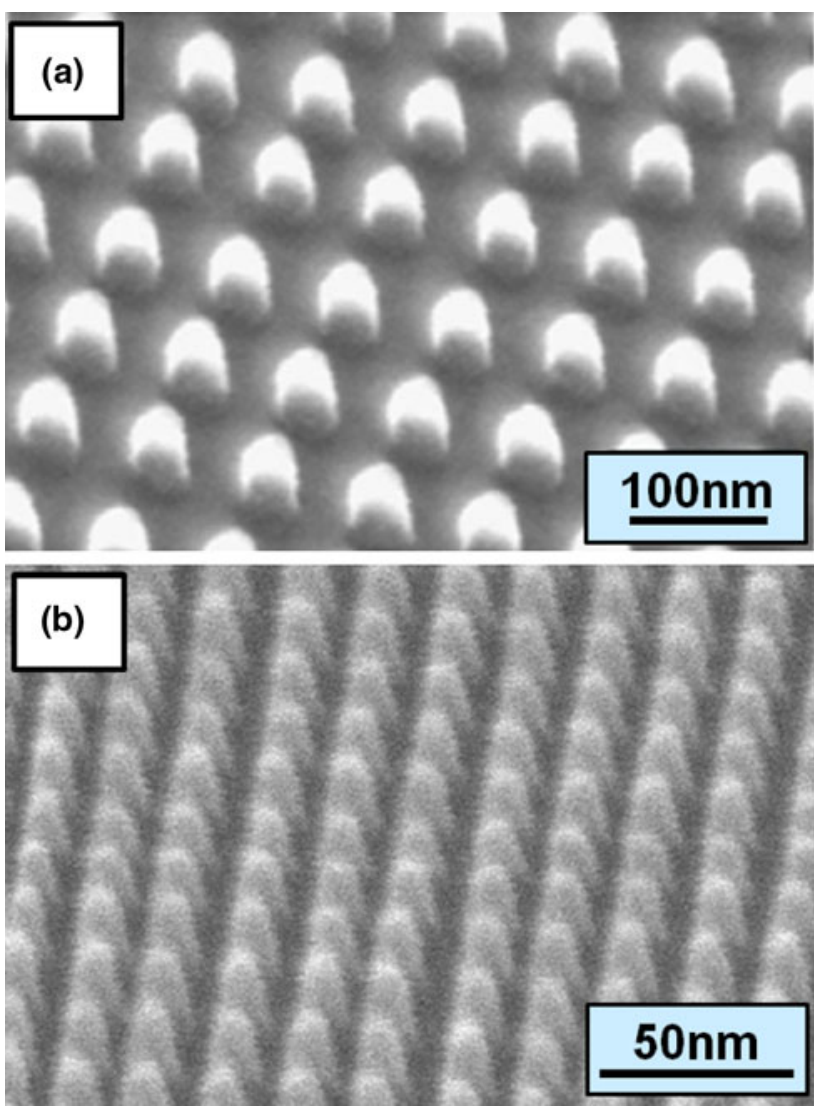

Fig. 3 a Oblique angle SEM images showing topographical features of bit patterned media $\left[30 \mathrm{~nm}\right.$ diameter bit array $\left(\sim 0.18 \mathrm{Tbit} / \mathrm{in}^{2}\right.$ density)] and (b) $10 \mathrm{~nm}$ diameter bit array (1.6 Tbit/in. ${ }^{2}$ density)

Figure $4 \mathrm{a}$ shows the media before planarization and Fig. $4 \mathrm{~b}$ after planarization. The periodicity, diameter and height of the pillars are 100, 40 and $40 \mathrm{~nm}$, respectively. As shown in the inset of Fig. 4a, the peak-to-valley height of the patterned media is $55 \mathrm{~nm}$. This value is identical to the sum of the height of the pillars $(40 \mathrm{~nm})$ and the magnetic layer $(15 \mathrm{~nm})$ while the peak-to-valley height measured on the planarized BPM (Fig. 4b) is $15 \mathrm{~nm}$. Clearly, only the magnetic layer on top of the pillars is exposed and the trench space is filled with HSQ, a nonmagnetic material. The BPM manufactured on a Si substrate coupon using e-beam lithography is far too small to test slider flyability.

In order to optimize the condition of planarization and test slider flyability, it is necessary to use a real size disk such as, for instance, a $65 \mathrm{~mm}$ (2.5 in.) disk. However, research and development toward manufacturing of such a large area with uniform nano-islands is still in progress. In addition, it would be costly and time consuming in the laboratory to fabricate such a large disk with precise nanopatterning for flyability testing. As an alternative, we created a nano-patterned disk with a distribution of nano-islands using a metal island mask produced with the "ball-up" process described previously. A SEM image of 
Fig. 4 SEM (upper) and AFM (lower) images of a before planarization and $\mathbf{b}$ after planarization of bit patterned islands. The insets in the AFM images show line scan profiles
Fig. 5 SEM images of a patterned pillars with $\mathrm{Ag}$ "ball-up" method, b patterned pillars planarized with HSQ, c planarized pillars by RIE etching, and $\mathbf{d}$ planarized pillars with recess by RIE overetching. The inset represents a schematic diagram

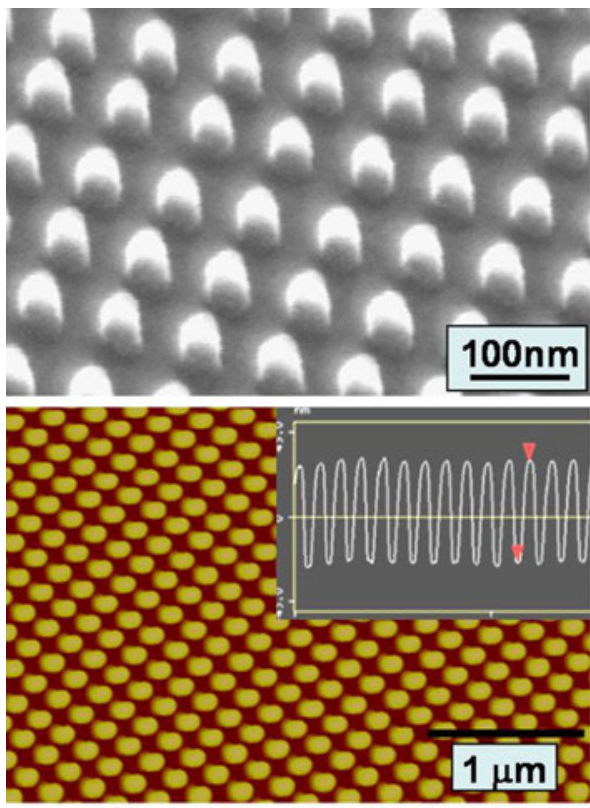

(a) Before planarization

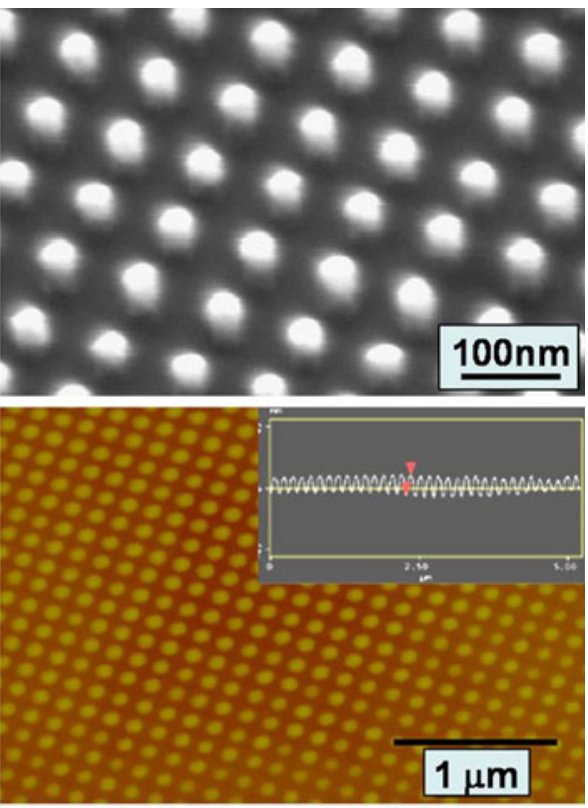

(b) After planarization
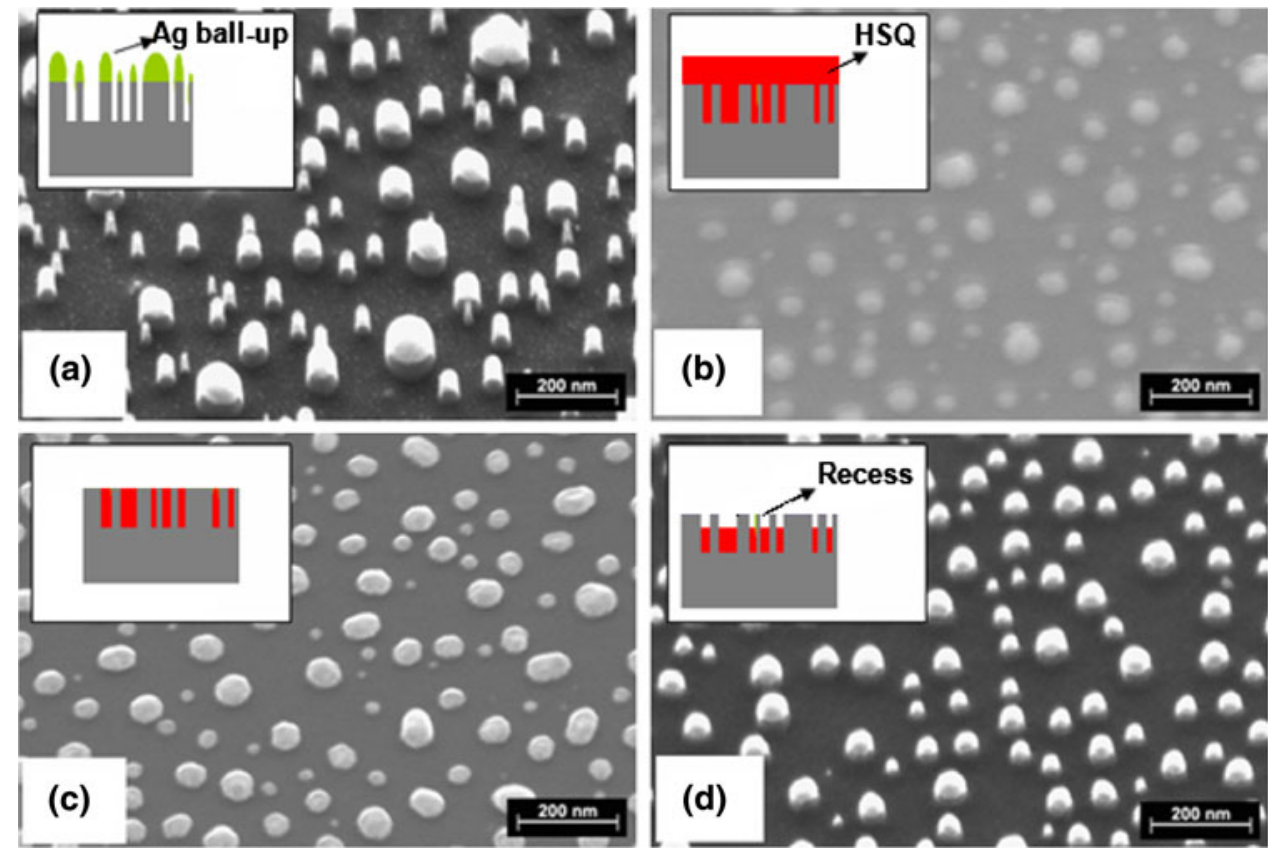

typical nano-pillars obtained in this way is shown in Fig. 5a. The height of the pillars created on a glass substrate is approximately $40 \mathrm{~nm}$. This height is considered large enough to avoid magnetic interactions of the recording media material deposited in the valleys with the material deposited on the top of the pillars. In the next step we deposited HSQ for planarization of the media. Figure $5 \mathrm{~b}$ shows nano pillars with spin coated HSQ resist. We observe that all nano-pillars with $\mathrm{Ag}$ islands are buried in the HSQ. It should also be noted that the surface of planarized BPM with HSQ is very "smooth" as indicated by AFM analysis, similar to the roughness of the glass substrate prior to planarization. In contrast, the surface of BPM planarized with sputter-coated $\mathrm{SiO}_{2}$ tends to yield topography similar to the underlying patterned substrate. (Hattori et al. 2004) After spin-coating, the surplus HSQ was etched back (Fig. 5b) by RIE. The time of the RIE can be controlled to fabricate BPM with desired recess depth. An 
image of a planarized bit patterned medium after backetching is shown in Fig. 5c. We observe that the trenches are completely filled and that only the Ag masks on the glass pillar are exposed. If the back etching time is optimized, a bit patterned medium with shallow recess topography is obtained as shown in Fig. 5d.

In preliminary tests we observed that HSQ did not provide sufficient mechanical strength to provide an acceptable tribological interface. It has been reported that the chemical structure of HSQ exhibits a ladder or cagelike arrangement which is mechanically "soft". This structure can be transformed into a mechanically stronger, $\mathrm{SiO}_{2}$-like network structure if HSQ is exposed to oxygen plasma. (Penaud et al. 2006; Kawamori et al. 2006) Therefore, prior to flyability testing, we have applied oxygen plasma irradiation to harden and improve the density of the planarized HSQ. The BPM structure processed in this way exhibited much better flyability.

To investigate the flyability, a laser Doppler vibrometer (LDV) was used to measure the flying height fluctuations of the slider on the disk. In this experiment, we used a slider, which was designed to fly at a flying height of $11 \mathrm{~nm}$ on a smooth disk at a velocity of $22 \mathrm{~m} / \mathrm{s}$. Knigge et al. (2008) have shown that the flying height loss of magnetic recording sliders on BPM follows the empirical equation
$\Delta F H=d * \frac{\mathrm{A}_{\mathrm{r}}(\text { recessed area })}{\mathrm{A}_{\mathrm{t}}(\text { total area })}$

where $\mathrm{d}$ is the height of an exposed pillar.

Using this equation, we have estimated the flying height loss of typical sliders on BPM. The area ratio of recessed area to total area in our media was found to be 0.45 by averaging the values for several locations of $1 \mu \mathrm{m} \times 1 \mu \mathrm{m}$ sampling area, using a commercially available analysis software and calculated the recess area (=total area - land area). The flying height loss is proportional to this ratio, according to the Eq. 1. On a smooth, un-patterned disk, good flyability was observed as shown in Fig. 6a. However, on un-planarized bit patterned disks with $40 \mathrm{~nm}$ high bits, the slider did not fly at all. After planarization of the disk, the slider showed excellent flying characteristics (Fig. 6b). In particular, on the planarized disk the flying height fluctuations of the slider were similar to those for a smooth un-patterned media (Fig. 6a). However, when the depth of the HSQ recess relative to the pillar top surface was increased to 10 or $20 \mathrm{~nm}$, respectively, by controlling the degree of RIE of deposited $\mathrm{SiO}_{2}$ planarization material, the interactions between slider and disk were found to increase substantially. This can be seen clearly from the flying height modulation data shown in Fig. $6 \mathrm{c}$ and d, i.e., the peak to peak values of fluctuations on BPM with recess
Fig. 6 Fluctuations of flying height of sliders on a unpatterned smooth media, b planarized BPM with no recess, $\mathbf{c}$ planarized BPM with $10 \mathrm{~nm}$ recess, and d planarized BPM with $20 \mathrm{~nm}$ recess
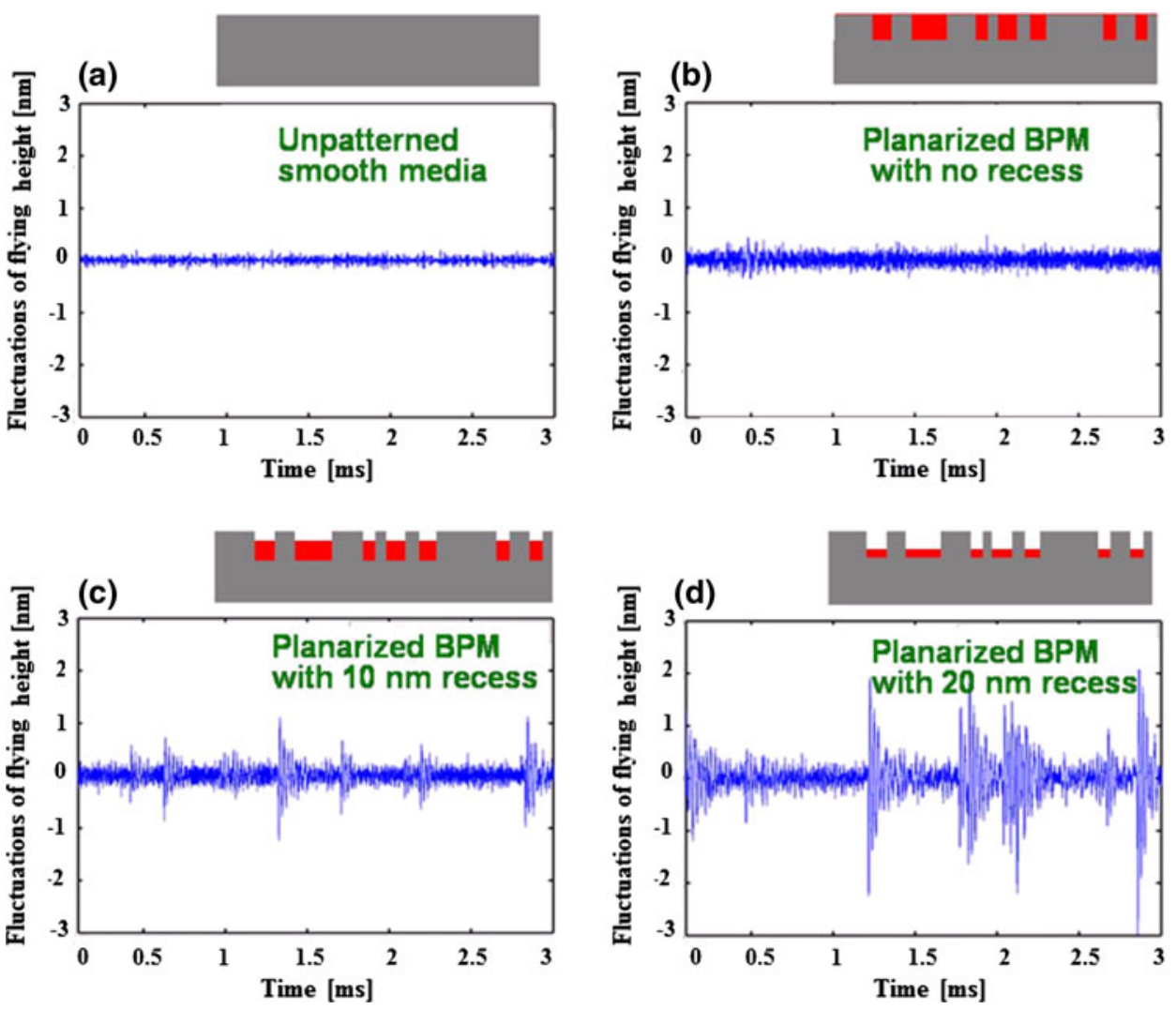
Fig. 7 Comparison of frequency spectra a on unpatterned smooth disk, $\mathbf{b}$ on planarized BPM with no recess, c on planarized BPM with $10 \mathrm{~nm}$ recess, and $\mathbf{d}$ on planarized BPM with $20 \mathrm{~nm}$ recess
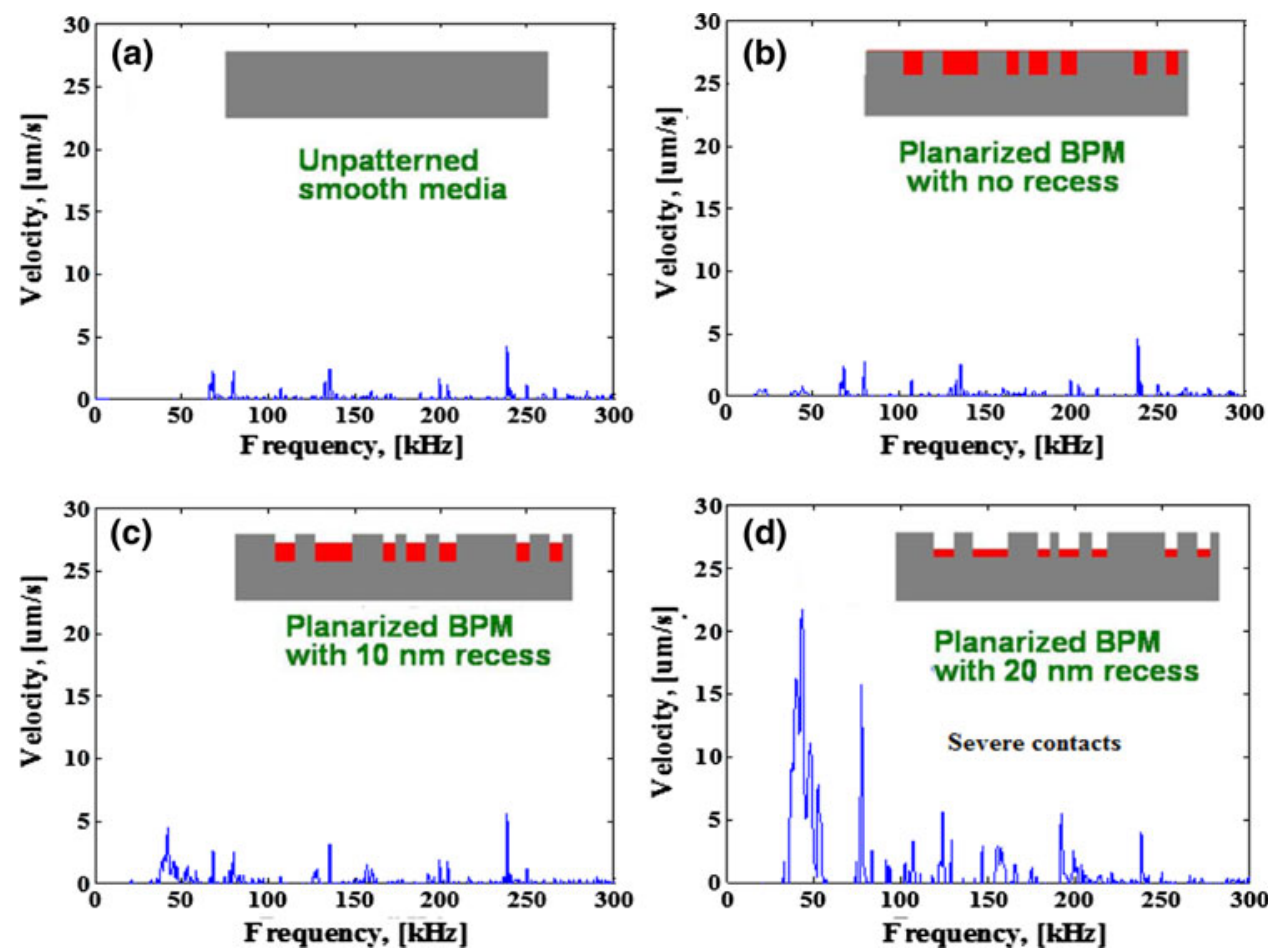

Table 1 Flying height on BPM with various recess depths after planarization

\begin{tabular}{ll}
\hline Recess depth $(\mathrm{nm})$ & Flying height $(\mathrm{nm})$ \\
\hline Zero & 11 \\
10 & 5.5 \\
20 & 0 \\
\hline
\end{tabular}

of 10 and 20 and 1 and $3 \mathrm{~nm}$, respectively. Figure 7 shows frequency spectra with respect to different planarization conditions. As can be seen from Fig. 7c, additional frequencies are observed in the range from 30 to $100 \mathrm{kHz}$, i.e., increased fluctuations of the slider occur. Clearly Fig. 7d indicates that severe contacts occurred during flyability testing on planarized BPM with $20 \mathrm{~nm}$ recess. These results show good agreement with previous flying height data (Table 1). Table 1 indicates the flying height of sliders with various recess depths after planarization. It is apparent from our study that planarization of the nanoscale patterned recording media has significantly improved the flyability of the head/disk interface.

\section{Summary and conclusions}

We have fabricated nanoscale BPM by e-beam lithography and RIE. Planarization of BPM was performed using spin coating of HSQ. Plasma treatment was used to mechanically strengthen the HSQ resist, and etch back was used to remove excess material. A large diameter $(65 \mathrm{~mm})$ disk with nano-topographical features was fabricated using a "ball-up" technique and RIE. The flyability of magnetic recording sliders was then evaluated for planarized disks with HSQ and compared with partially planarized disks. HSQ-planarized disks exhibited significantly improved head flyability, similar to that of smooth glass disk surfaces, indicating that the read/write head flyability problem on nano patterned, high-density recording media can be substantially mitigated.

Acknowledgments This research was supported by CMRR (Center for Magnetic Recording Research) at UC San Diego, NSF-CMMI, Nanomanufacturing Division, Grant No. 0856674, CNMT Grant No. 05K1501-01210 under the 21st Century Frontier R\&D Programs, Ministry of Science and Technology, Korea, and National Research Foundation (NRF) grant through World Class University Program (R33-2008-000-10025-0).

Open Access This article is distributed under the terms of the Creative Commons Attribution Noncommercial License which permits any noncommercial use, distribution, and reproduction in any medium, provided the original author(s) and source are credited.

\section{References}

Hattori K, Ito K, Soeno Y, Takai M, Matsuzaki M (2004) Fabrication of discrete track perpendicular media for high recording density. IEEE Trans Magn 40:2510-2515

Kawamori M, Nakamatsu K, Haruyama Y, Matsui S (2006) Effect of oxygen plasma irradiation on hydrogen silsesquioxane nanopatterns replicated by room-temperature nanoimprinting. Jpn J Appl Phys 45:8994-8996 
Kitade Y, Komoriya H, Maruyama T (2004) Patterned media fabricated by lithography and argon-ion milling. IEEE Trans Magn 40:2516-2518

Knigge BE, Bandic ZZ, Kercher D (2008) Flying characteristics on discrete track and bit-patterned media with a thermal protrusion slider. IEEE Trans Magn 44(11):3656-3662

Penaud J, Fruleux F, Dubois E (2006) Transformation of hydrogen silsesquioxane properties with RIE plasma treatment for advanced multiple-gate MOSFETs. Appl Surf Sci 253:395-399

Richter HJ, Dobin AY, Heinonen O, Gao KZ, Veerdonk RJMvd, Lynch RT, Xue J, Weller D, Asselin P, Erden MF, Brockie RM
(2006) Recording on bit-patterned media at densities of 1Tb/in2 and beyond. IEEE Trans Magn 42(10):2255-2260

Shaw JM, Rippard WH, Russek SE, Reith T, Falco CM (2007) Origins of switching field distributions in perpendicular magnetic nanodot arrays. J Appl Phys 101:023909-023912

Terris BD, Thomson T (2005) Nanofabricated and self-assembled magnetic structures as data storage media. J Phys D Appl Phys 38:R199-R222

Thomson T, Hu G, Terris BD (2006) Intrinsic distribution of magnetic anisotropy in thin films probed by patterned nanostructures. Phys Rev Lett 96:257204-257207 